\title{
Interaction of daylength and lactation in the control of pelage development and nest-building in female meadow voles (Microtus pennsylvanicus)
}

\author{
T. M. Lee, L. Smale, I. Zucker and J. Dark \\ Department of Psychology, University of California, Berkeley, CA 94720, U.S.A.
}

\begin{abstract}
Summary. Pregnancy and lactation inhibited moult into winter pelage in voles maintained in short daylengths; development of a winter pelage was, however, greatly accelerated once the short-day dams weaned their litters. The presumed elevation of prolactin titres during lactation appears to mask full development and expression of pelage changes induced by short daylengths. Nest-building behaviour, by contrast, was increased in response to short photoperiods and was further augmented during lactation and may thereby facilitate thermoregulation in short-day dams that do not develop a winter pelage.
\end{abstract}

\section{Introduction}

At 10 weeks after transfer from a long to a short photoperiod, meadow voles have a thick winter pelage and manifest increased nest building activity, decreased body weight and reduced food intake (Dark \& Zucker, 1983; Dark et al., 1983); males undergo testicular involution and females are less likely to become pregnant (Dark \& Zucker, 1983; Lee et al., 1987). This integrated set of changes probably facilitates survival under winter conditions (Dark \& Zucker, 1985).

In mink and Siberian hamsters the moult to a winter pelage induced by short daylengths is mediated by decreased circulating prolactin concentrations, and effects of daylength on pelage can be counteracted by exogenous prolactin (Duncan \& Goldman, 1984; Martinet et al., 1984).

Although most meadow voles are reproductively quiescent during fall and winter, a sizeable minority breeds between September and January (Getz et al., 1979; Christian, 1980). Prolactin secretion is probably elevated in these lactating females during the short days of winter. These voles face a dilemma: a decrease in prolactin secretion may be necessary for the moult into winter pelage, while increased prolactin secretion may be required for lactation. It seemed probable that the elevated prolactin concentrations associated with lactation could prevent or reverse the effects of short daylengths on the pelage. Furthermore, voles that are impregnated after developing a winter coat may, under the lactational stimulus of elevated prolactin secretion, prematurely moult into summer pelage. One purpose of these experiments was to evaluate how conflicting signals from short daylengths and lactation are resolved to regulate pelage growth and maintenance.

Nest-building behaviour, a thermoregulatory response that increases during pregnancy and lactation (Leon et al., 1978; Alberts \& Gubernick, 1983), is also stimulated in meadow voles by short photoperiods (Dark \& Zucker, 1983). The present experiments determined whether lactation and short daylengths have additive effects on nest building. That is, do lactating voles exposed to the short daylengths of winter compensate for a less developed winter pelage by building larger and perhaps more insulating nests? 


\section{Materials and Methods}

\section{Experiment 1}

Animals. Fifty-eight adult female voles (Microtus pennsylvanicus), all but two of them nulliparous, were maintained from birth in a room illuminated for $14 \mathrm{~h}$ per day. They were housed individually or in pairs in cages with Purina Diet No. 5015 and tap water available ad libitum.

Procedures. Beginning at Week 1, animals aged 60-180 days, were housed individually in cages in light-tight ventilated chambers and randomly assigned to illumination with fluorescent bulbs for $14 \mathrm{~h}$ daily (long days; LD; lights on $07: 00 \mathrm{~h}$ ) or $10 \mathrm{~h} /$ day (short days; SD; lights on $07: 00 \mathrm{~h}$ ). Room temperature was kept at $23 \pm 2^{\circ} \mathrm{C}$. A long-day male of proven fecundity was housed with each female during Week 3. After mating, sample size for each group was $13-16$ animals. Young were weaned at 3 weeks of age (Week 9). Females that failed to deliver litters after pairing with males constituted the comparison groups.

To measure nest building behaviour, cotton batting of known weight ( $\sim 30 \mathrm{~g}$ per animal) was placed on the cage lid of each animal for $24 \mathrm{~h}$ once per week for the 13 weeks of the experiment. The cotton remaining on the lid was reweighed during Week 2, and each of Weeks 4-13; measurements were not taken for Week 1 , which was an adaptation period, and during Week 3, when males were present in the cages. Nests were left in place until 2 days before the next scheduled nest-building measurement.

At weaning (Week 9), all lactating and unimpregnated females were lightly anaesthetized with halothane and hair depth from pelage surface to skin surface was measured to the nearest $0.1 \mathrm{~mm}$ with a micrometer (McClure $\&$ Porter, 1983), relative hair density was determined by weighing hair shaved from a $1 \mathrm{~cm}^{2}$ patch (Al-Khateeb \& Johnson, 1971), and guard hair and under hair lengths were measured to the nearest 0.1 mm (Dark \& Zucker, 1983; McClure \& Porter, 1983). All pelage measures were made on the posterior dorsal surface. For a detailed discussion of the importance of these pelage measures for thermoregulation see McClure \& Porter (1983) and Al-Khateeb \& Johnson (1971).

At 4 weeks after weaning (Week 13), all animals were given a lethal dosage of pentobarbitone sodium and pelage was measured as described above. The posterior dorsal surface was then shaved clean and a $2 \mathrm{~cm}^{2}$ patch of skin was removed and preserved in $10 \%$ formalin, embedded in paraffin wax, sectioned at $5 \mu \mathrm{m}$ and stained with haematoxylin and eosin. The mean number of follicles for 10 intact follicular bundles was determined microscopically for each animal.

\section{Experiment 2}

Animals and procedures. At Week 1, 56 adult nulliparous voles, maintained from birth in long days, were housed individually in cages and transferred to environmental chambers illuminated with long or short days. During Week 9, a fecund LD male was placed in the cage of each female in SD; LD females were not paired with males. After mating, sample size was 11,14 and 32 for LD, SD impregnated and SD unimpregnated groups, respectively. Comparison groups consisted of unmated LD females and SD females that failed to conceive. Young were weaned at 3 weeks of age (Week 15). Experiments 1 and 2 differ in the duration of the preadaptation period for the females to the short photoperiod before mating ( 2 versus 8 weeks in Exps 1 and 2, respectively).

Nest building behaviour, as described in Exp. 1, was measured during Weeks $2-8$ and 10-19. Pelage was measured at weaning (Week 15); final measures of pelage and a skin sample were obtained at Week 19. The depth and length of under hairs were measured during Week 9 , to provide an index of the response to short days before mating.

The animals from Exps 1 and 2 are those for which body weights and food intakes have been described (Lee $e t$ al., 1987).

\section{Statistical analyses}

Experiment 1. Nest size and pelage measures (depth, density, guard and under hair lengths) were analysed using a multivariate analysis of variance (MANOVA) which supplied a multivariate $F$ value and individual $F$ values of nest size for each measure of pelage for each week. When overall group differences existed, planned comparisons were performed between LD and SD unimpregnated females, LD and SD impregnated females and impregnated $v s$ unimpregnated females. Follicle number was analysed with ANOVA and planned comparisons as listed above.

Experiment 2. Data were analysed as described above. In addition, SD animals from the two experiments were compared using a MANOVA to assess effects of 2 versus 8 weeks of preadaptation to the short photoperiod. Results are reported as significant for probabilities $\leq 0.05$.

\section{Results}

\section{Experiment 1}

Nest weight. Nest building was influenced by photoperiod and by lactation (Fig. 1); in both 

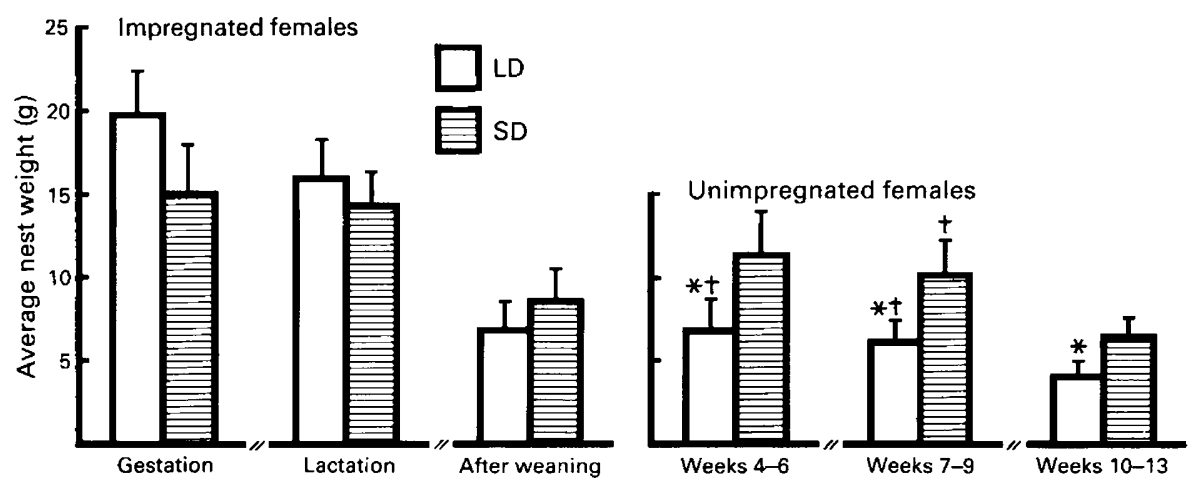

Fig. 1. Nest material gathered by impregnated or unimpregnated voles housed in long (LD) or short (SD) daylengths for Exp. $1 .{ }^{*} P<0.05$, compared with short daylength values. $\nmid P<0.05$ compared with impregnated animals within the same photoperiod.
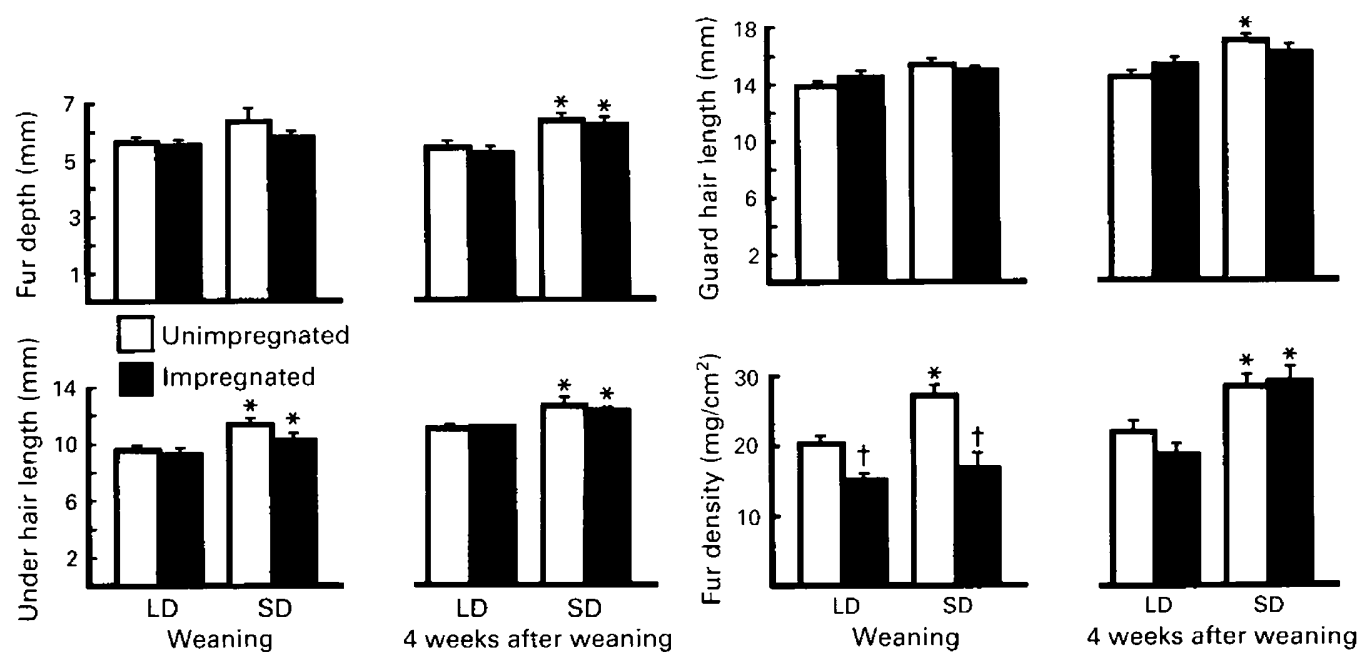

Fig. 2. Fur characteristics in unimpregnated or impregnated voles housed in long (LD) or short (SD) daylengths. Measurements were made after 9 weeks (weaning) and 13 weeks (4 weeks after weaning) for all animals. ${ }^{*} P<0.05$, compared with LD values. $\dagger P<0.05$, compared with animals within the same photoperiod.

photoperiods pregnant and/or lactating females built heavier nests than did unimpregnated voles. Nest size did not differ at any time between LD and SD impregnated animals. Unimpregnated SD voles built significantly larger nests than did their LD counterparts during Weeks 4-13. During Weeks 7-9 nest size was significantly smaller for the SD unimpregnated group than for the lactating LD or SD voles. Across the 13 weeks of testing groups ranked as follows with respect to nest size: LD unimpregnated $<$ SD unimpregnated $\leq$ SD and LD lactating animals.

Pelage. After 9 weeks of treatment, fur density and under hair length were significantly increased in SD as compared to LD unimpregnated females (Fig. 2); by 13 weeks these measures, as well as fur depth and guard hair length, were significantly greater in SD than LD unimpregnated animals (Fig. 2). At 13 weeks the number of hair follicles per follicular bundle also was significantly 
greater for SD than LD unimpregnated animals $(\mathrm{SD}=7 \cdot 6 \pm 0 \cdot 3, \mathrm{LD}=6.9 \pm 0 \cdot 2 ; t=2 \cdot 09$, $P<0.05)$.

Lactation counteracted the effects of short daylengths on pelage characteristics. At weaning (Week 9), LD and SD dams did not differ with respect to fur density, depth or guard hair length; only under hair length was greater in SD than LD dams (Fig. 2). At weaning SD lactating females had less dense fur and shorter under hairs than did SD unimpregnated animals (Fig. 2). LD lactating females also had less dense fur than did the unimpregnated LD comparison females. At 4 weeks after weaning (Week 13) the effects of photoperiod were evident in SD dams: 3 of 4 pelage characteristics were significantly increased in SD as compared to LD dams and values for SD dams were equivalent to those of SD unimpregnated animals (Fig. 2). Follicle number/follicular bundle was equal in LD dams and LD unimpregnated females $(6 \cdot 8 \pm 0 \cdot 3,6.9 \pm 0 \cdot 2$, respectively). Follicle number for SD dams $(7 \cdot 4 \pm 0.4)$ was intermediate between LD and SD $(7 \cdot 6 \pm 0 \cdot 3)$ values for unimpregnated females and did not differ significantly from either.

Table 1. Nest material (g) gathered by impregnated or unimpregnated voles preadapted to short daylengths for 8 weeks

\begin{tabular}{|c|c|c|c|}
\hline Week & $\begin{array}{c}\text { LD- } \\
\text { unimpregnated }\end{array}$ & $\begin{array}{c}\text { SD- } \\
\text { unimpregnated }\end{array}$ & $\begin{array}{c}\text { SD- } \\
\text { lactating }\end{array}$ \\
\hline 3 & $4 \cdot 2 \pm 1 \cdot 2$ & $6 \cdot 9 \pm 1 \cdot 0$ & $6.8 \pm 1.8$ \\
\hline 5 & $3.6 \pm 0.9 *$ & $7 \cdot 2 \pm 1 \cdot 1$ & $4.4 \pm 0.9$ \\
\hline 7 & $2 \cdot 1 \pm 0.6^{*}$ & $8 \cdot 6 \pm 1 \cdot 1$ & $5.5 \pm 0.9$ \\
\hline 10 (pregnant) & $5 \cdot 0 \pm 1 \cdot 5^{*}$ & $10.7 \pm 1.4$ & $12 \cdot 2 \pm 2 \cdot 7$ \\
\hline 11 (pregnant) & $3.4 \pm 1 \cdot 1^{*}$ & $13 \cdot 2 \pm 1 \cdot 4$ & $12.5 \pm 1.6$ \\
\hline 13 (lactating) & $4.9 \pm 1.5^{*}$ & $11 \cdot 0 \pm 1 \cdot 2$ & $18.9 \pm 3.0 *$ \\
\hline 15 (lactating) & $8.5 \pm 1.8^{*}$ & $12.9 \pm 2.8$ & $17.7 \pm 2.8^{*}$ \\
\hline 17 (after weaning) & $3 \cdot 3 \pm 0.7^{*}$ & $6.7 \pm 1.0$ & $3.4 \pm 0.6$ \\
\hline 19 (after weaning) & $3.0 \pm 0.7^{*}$ & $8.4 \pm 1 \cdot 0$ & $6.6 \pm 0.9$ \\
\hline
\end{tabular}

Values are mean \pm s.e.m.

*Differs significantly from SD-unimpregnated value $(P<0.01)$.

Table 2. Pelage measures for voles preadapted to short daylengths for 8 weeks

\begin{tabular}{|c|c|c|c|}
\hline & $\begin{array}{c}\text { LD- } \\
\text { unimpregnated }\end{array}$ & $\begin{array}{c}\text { SD- } \\
\text { unimpregnated }\end{array}$ & $\begin{array}{c}\text { SD- } \\
\text { lactating }\end{array}$ \\
\hline \multicolumn{4}{|l|}{ Before test (Week 9) } \\
\hline Depth $(\mathrm{mm})$ & $5 \cdot 4 \pm 0 \cdot 1^{*+}$ & $6 \cdot 9 \pm 0 \cdot 2$ & $6 \cdot 4 \pm 0.3$ \\
\hline Under hair (mm) & $10.4 \pm 0.5^{*} \dagger$ & $12 \cdot 2 \pm 0.4$ & $11.4 \pm 0.5$ \\
\hline \multicolumn{4}{|l|}{ Weaning (Week 15) } \\
\hline Depth $(\mathrm{mm})$ & $6.9 \pm 0.2^{*} \dagger$ & $7 \cdot 4 \pm 0 \cdot 1$ & $6.7 \pm 0.2^{*}$ \\
\hline Under hair (mm) & $10 \cdot 2 \pm 0.4^{*} \dagger$ & $12 \cdot 2 \pm 0.3$ & $11.5 \pm 0.3$ \\
\hline Guard hair (mm) & $14.8 \pm 0.4^{*}+$ & $17 \cdot 3 \pm 0 \cdot 3$ & $16.6 \pm 0.3$ \\
\hline Density $\left(\mathrm{mg} / 1 \mathrm{~cm}^{2}\right)$ & $23 \cdot 3 \pm 2 \cdot 2^{*}$ & $34 \cdot 6 \pm 2 \cdot 1$ & $28 \cdot 0 \pm 2 \cdot 4^{*}$ \\
\hline \multicolumn{4}{|l|}{ Final (Week 19) } \\
\hline Depth $(\mathrm{mm})$ & $5 \cdot 8 \pm 0 \cdot 1^{* \dagger}$ & $6.6 \pm 0.1$ & $6.7 \pm 0.1$ \\
\hline Under hair (mm) & $11 \cdot 2 \pm 0.4^{*} \dagger$ & $12.5 \pm 0.2$ & $12.4 \pm 0.3$ \\
\hline Guard hair (mm) & $16 \cdot 2 \pm 0.4^{*} \dagger$ & $17.5 \pm 0.2$ & $17 \cdot 5 \pm 0.4$ \\
\hline Density $\left(\mathrm{mg} / 1 \mathrm{~cm}^{2}\right)$ & $23.6 \pm 1.5^{*} \dagger$ & $32.9 \pm 1.6$ & $29 \cdot 1 \pm 2 \cdot 3$ \\
\hline Follicle number & $7 \cdot 4 \pm 0.3^{*}$ & $8.2 \pm 0.2$ & $7.6 \pm 0.3$ \\
\hline
\end{tabular}

Values are mean \pm s.e.m.

*Differs significantly from values for SD-unimpregnated voles, $P<0.05$.

$\dagger$ Differs significantly from values for SD-lactating voles, $P<0 \cdot 05$. 


\section{Experiment 2}

Nest size. Nest size increased by Week 5 and remained elevated through Week 19 in SD voles (Table 1). During lactation SD animals increased their nest size to values that exceeded those of SD unimpregnated animals (Weeks 13-15). Lactation therefore stimulated an increase in nest-building over and above that produced by short daylengths before impregnation.

Pelage. Pelage depth and under hair length were significantly increased for both groups of SD animals as compared to LD unimpregnated animals at Week 9 (before mating; Table 2). At the time of weaning (Week 15), the LD and SD unimpregnated animals differed on all pelage measures (Table 2). Of greatest interest, fur depth and density of SD lactating females was reduced at weaning relative to that of SD unimpregnated animals (Table 2).

At 4 weeks after weaning (Week 19), pelage measurements did not differ between SD impregnated and unimpregnated animals; SD and LD unimpregnated animals continued to differ on all measures (Table 2). As in Exp. 1, hair follicle number/follicular bundle was significantly greater in SD than in LD unimpregnated voles and the lactating animals were intermediate (Table 2).

\section{Discussion}

Short daylengths induce changes in nest-building and pelage in female meadow voles and thereby probably facilitate thermoregulation by decreasing heat loss at low ambient temperatures. Nest size was increased after 4 weeks and fur was deeper, longer and more dense after 8-9 weeks of short-day treatment. Pregnancy and lactation were associated with increased nest building and inhibition of hair growth in long and short photoperiods.

Short daylengths facilitated an approximate doubling of nest size. Pregnant and lactating animals increased nest building over and above increments induced by short daylengths alone. Increased nest size presumably is adaptive in warm or cool temperatures in helping maintain a narrow range of temperatures for the altricial litter (Leon et al., 1978; Alberts \& Gubernick, 1983).

Pregnancy and lactation inhibited pelage growth in animals maintained in short daylengths for 2 weeks before mating (Exp. 1); in fact, hair density was reduced in LD- and SD-impregnated females at the time of weaning. This observation is consistent with data from Siberian hamsters (Duncan \& Goldman, 1984), Limousine rams (Allain et al., 1986), mink (Martinet et al., 1984) and mice (Fraser \& Nay, 1953). The high prolactin concentrations of lactation appeared to prevent hair growth in animals kept in short daylengths. After the young were weaned, SD females developed a winter pelage within 4 weeks. The latency of this response was much shorter than that of nonlactating females and suggests that hair follicle development occurred before weaning, but hair growth was prevented by the hormonal milieu of lactation. Apparently, as in mice, the decrease in the dam's prolactin concentrations at weaning allows reinstatement of hair growth (Fraser \& Nay, 1953).

The results of Exp. 2 support this hypothesis: moult to the longer, denser winter pelage was well under way in short-day females before mating, but appeared to have been arrested at this stage upon impregnation. Within 4 weeks of weaning of their young, short-day dams completed hair growth to the same pelage length, density and depth that non-mated animals attain between Weeks 8 and 19 in the short photoperiod. At 4 weeks after weaning, the number of hair follicles per follicular bundle in SD dams was intermediate between that of LD and SD unimpregnated animals and not different from either. Again, this supports the hypothesis that pelage development proceeds after impregnation, but its full development and expression are masked by the hormonal milieu of pregnancy/lactation.

Pregnancy and lactation may compromise thermoregulation in voles in which winter pelage preparation has yet to begin (Exp. 1) or is in progress (Exp. 2). Increases in nest size during lactation may ameliorate the negative consequences of decreases in pelage length, density and depth. 
Since the primary effect of lactation is to halt new hair growth, the animals most at risk would be those undergoing repeated pregnancies as a result of post-partum matings during late summer and autumn. Voles breeding in February/March would already have a winter pelage and would not face this problem.

In some populations, meadow voles are pregnant or lactating in every month of the year (Christian, 1980); others have sizable breeding spurts in mid-winter, but pregnant females are not found in autumn (Christian, 1978, 1980; Mihok, 1985). It remains possible that few females produce litters at the time the winter moult is in progress, and the potential negative consequences of lactation on thermoregulation are rarely manifested. Nevertheless, voles breeding in short photoperiods, once the young are weaned, moult into winter pelage very rapidly compared to unmated animals.

We thank Lisa Higa and Chris Tuthill for technical assistance and Darlene Frost and Paul Haller for preparing and photographing the illustrations. This research was supported by NIH grant HD-02982, postdoctoral fellowship HD-06461 and a NIMH traineeship MH-15860.

\section{References}

Alberts, J.R. \& Gubernick, D.J. (1983) Reciprocity and resource exchange. A symbiotic model of parentoffspring relations. In Symbiosis in Parent-Offspring Interactions, pp. 7-44. Eds L.A. Rosenblum \& H. Moltz. Plenum Press, New York.

Al-Khateeb, A. \& Johnson, E. (1971) Seasonal changes of pelage in the vole (Microtus agrestis) I. Correlation with changes in the endocrine glands. Gen. comp. Endocr. 16, 217-228.

Allain, D., Ravault, J.P., Panaretto, B.A. \& Rougeout, J. (1986) Effects of pinealectomy on photoperiodic control of hair follicle activity in the Limousine ram: possible relationships with plasma prolactin levels. $J$. Pineal Res. 3, 25-32.

Christian, J.J. (1978) Neurobehavioral endocrine regulation of small mammal populations. In Populations of Small Mammals Under Natural Conditions, pp. 143-158. Ed. D. P. Snyder. Pymatuning Laboratory of Ecology, University of Pittsburgh.

Christian, J.J. (1980) Regulation of annual rhythms of reproduction in temperate small rodents. In Testicular Development, Structure, and Function, pp. 367-380. Eds A. Steinberger \& E. Steinberger. Raven Press, New York.

Dark, J. \& Zucker, I. (1983) Short photoperiods reduce winter energy requirements of the meadow vole, Microtus pennsylvanicus. Physiol. Behav. 31, 699-702.

Dark, J. \& Zucker, I. (1985) Seasonal cycles in energy balance: regulation by light. Ann. N.Y. Acad. Sci. 453, 170-181.

Dark, J., Zucker, I. \& Wade, G.N. (1983) Photoperiodic regulation of body mass, food intake and reproduction in meadow voles. Am. J. Physiol. 245, R334-R338.
Duncan, M.J. \& Goldman, B.D. (1984) Hormonal regulation of the annual pelage color cycle in the Djungarian hamster, Phodopus sungorus, II. Role of prolactin. J. exp. Zool. 230, 97-103.

Fraser, A.S. \& Nay, T. (1953) Growth of the mouse coat. II. Effect of sex and pregnancy. Aust. J. biol. Sci. 6, $645-656$.

Getz, L.L., Verner, L., Cole, F.R., Hofman, J.E. \& Avalos, D.E. (1979) Comparisons of population demography of Microtus ochrogaster and Microtus pennsylvanicus. Acta theriol. 24, 319-349.

Lee, T.M., Smale, L., Zucker, I. \& Dark, J. (1987) Role of photoperiod during pregnancy and lactation in the meadow vole, Microtus pennsylvanicus. J. Reprod. Fert. 81, 337-342.

Leon, M., Croskerry, P.G. \& Smith, G.K. (1978) Thermal control of mother-young contact in rats. Physiol. Behav. 21, 793-811.

Martinet, L., Allain, D. \& Weiner, C. (1984) Role of prolactin in the photoperiodic control of moulting in the mink (Mustela vison). J. Endocr. 103, 9-15.

McClure, P.A. \& Porter, W. (1983) Development of insulation in neonatal cotton rats (Sigmodon hispidus). Physiol. Zool. 56, 18-32.

Mihok, S. (1984) Life history profiles of boreal meadow voles (Microtus pennsylvanicus). In Winter Ecology of Small Mammals, Carnegie Museum of Natural History Special Publication, Vol. 10, pp. 91-102. Ed. J.F. Merritt, Carnegie Museum of Natural History, Pittsburgh. 